\title{
Sensitivity, probabilistic and stochastic analysis of the thermo-piezoelectric phenomena in solids by the stochastic perturbation technique
}

\author{
Marcin Kamiński • Alberto Corigliano
}

Received: 23 May 2010 / Accepted: 16 June 2011 / Published online: 12 July 2011

(C) The Author(s) 2011. This article is published with open access at Springerlink.com

\begin{abstract}
The main aim here is to present the application of the generalized stochastic perturbation technique to thermo-piezoelectric analysis of solid continua. The general $n$th order Taylor series representation for all random input parameters and the state functions is employed to formulate the coupled thermo-electro-elasticity equilibrium equations of the additional order; a determination of any probabilistic moments and characteristics is described; the discretization of the problem in terms of the Stochastic perturbation-based Finite Element Method is also provided. Since this expansion includes the lowest order partial derivatives, the structural sensitivity analysis using direct differentiation is performed at the same time with probabilistic modeling contrasted with the Monte-Carlo simulation results. The probabilistic approach is extended here towards an accounting for the stochastic ageing processes, which appear frequently in aggressive external environments and under dynamic excitation. The two parametric stochastic
\end{abstract}

\section{Kamiński $(\varangle)$}

Faculty of Civil Engineering, Architecture and

Environmental Engineering, Technical University of Łódź,

Al. Politechniki 6, 90-924 Łódź, Poland

e-mail: Marcin.Kaminski@p.lodz.pl

\author{
A. Corigliano \\ Dipartimento di Ingegneria Strutturale, Politecnico \\ di Milano, Piazza Leonardo da Vinci, 32, 20133 Milano, \\ Italy \\ e-mail: alberto.corigliano@polimi.it
}

process with Gaussian initial value and ageing velocity is tested for this purpose. The entire procedure is tested on the example of the thermo-electro-elastic pulsation of the beam modeled analytically using the symbolic software MAPLE, where polynomial approximations, design sensitivities, probabilistic moments and their histories are computed and visualized.

Keywords Coupled field problems · Stochastic perturbation method - Thermo-piezoelectricity . Reliability analysis · MEMS devices · Monte-Carlo simulation

\section{Introduction}

Probabilistic modeling of the MEMS devices belongs to wider class of such problems in computational mechanics, where the application of the Monte-Carlo simulation technique is very costly. This is primarily due to the multi-scale and multi-component character $[1,3,4]$ of such devices as well as to the thermoelectro-magneto-elastic couplings of quite different physical fields acting on them, which significantly enlarges the effective total number of degrees of freedom in the model; so that, the alternative computational techniques are considered and developed. Similarly to the other multiscale problems, the homogenization method may be applied $[9,15]$, which reduces significantly the number of various scales to the single or two, where hybrid numerical approaches link FEM computations with the analytical formulas for 
the overall effective characteristics of the entire structure.

One of such an alternative is the stochastic perturbation technique $[7,8]$, where hierarchical equations of a deterministic nature (quite similar to the original deterministic problem) are formed and solved. It needs an initial specification of the random input variable(s) of the problem, an assumption on its (their) probability density function(s) and a definition of those distribution(s) basic parameters. It is known from the Second Order Second Moment (SOSM) approach invented for and effectively used in structural analysis [8] that lower order techniques have a strong limitation to the case studies with small input random dispersion, quite contrary to the $n$th generalized perturbation method. Since the higher order equations include a series of partial derivatives (of the increasing order) of all input parameters and the structural response with respect to the random input variables, the essential part of this method is determination of those derivatives. The final calculation of the structural response probabilistic moments proceeds quite straightforwardly from the perturbation-based equations including previously determined various orders solutions and the probabilistic moments of the random input. Sometimes, where derivation of the partial derivatives may result in uncontrolled progress of the computational error, some approximation techniques are used to recover the function of the structural response under consideration with respect to the randomized input variable. The last opportunity excludes the necessity of the full access to the source code of the program being especially attractive for the commercial codes users, however needs the several solutions around the mean values of the random input parameters.

The presentation of this method starts from the general description of the basic probabilistic moments and characteristics determination (classical definitions and perturbation-based formulas), then the stochastic perturbation-based variational formulations are provided together with the Stochastic Finite Element Method (SFEM) equations to be formed and solved in the general case. A numerical illustration is provided on the example of the eigenproblem of the elastic beam with random length defined as the Gaussian random variable (and also nonstationary process). This problem is solved analytically using the computer algebra system MAPLE, v. 13, where all necessary prob- abilistic moments of the resulting pulsation are determined; the parametric studies with respect to the beam length expected value and its coefficient of variation are provided thanks to the usage of the symbolic computations. Moreover, the symbolic calculus usage enables one to take into account directly the perturbation parameter $\varepsilon$, which is absolutely impossible into the classical SFEM analysis, and then probabilistic convergence of the particular moments may be relatively easily verified in a pure analytical way as a simple limiting procedure on quasi-polynomial expressions. It would be especially valuable for the probability distribution functions other than the Gaussian, where higher order probabilistic moments cannot be defined uniquely using the first two, so that the convergence verification cannot proceed directly-for most of the distributions the moments generating functions exist only (with the exception to the lognormal distribution having recursive direct expressions for any moments). Let us finally note that further usage of the stochastic technique described below may directly lead to the direct numerical determination of the reliability index for the MEMS devices defined in a simple case as the ratio of the expected value to the standard deviation of the limit function, usually defined as the difference between the actual and the allowable state function values (like stresses, displacements, vibration frequencies or temperatures, for instance). We compare this approach with the classical statistical estimation results obtained from the Monte-Carlo simulation and it is done as the function of the input coefficient of variation for the beam length. As it is documented, lower order moments like expectations and variances are very close to each other, while third and fourth central probabilistic moments-only for the values of $\alpha$ smaller than 0.15 . Finally, we study probabilistic moments of the thermopulsation frequency in the case of the length of the nanostructure subjected to the stochastic non-stationary fluctuations. It could be the starting point to and the very beneficial in the full stochastic coupled problems reliability analysis of the MEMS devices.

\section{Uncertainty analysis}

2.1 The generalized stochastic perturbation technique

Let us consider the input random variable of the problem by $b(\omega)$, its probability density function as $g(b)$. 
The expected value of this variable may be expressed by $[7,8]$

$$
E[b]=\int_{-\infty}^{+\infty} b g(b) d b,
$$

while the central $m$ th probabilistic moment as

$\mu_{m}(b)=\int_{-\infty}^{+\infty}(b-E[b])^{m} g(b) d b$.

The coefficient of variation, asymmetry, flatness and kurtosis are introduced in the form

$$
\begin{aligned}
& \alpha(b)=\frac{\sqrt{\operatorname{Var}(b)}}{E[b]}=\frac{\sigma(b)}{E[b]}, \quad \beta(b)=\frac{\mu_{3}(b)}{\sigma^{3}(b)}, \\
& \gamma(b)=\frac{\mu_{4}(b)}{\sigma^{4}(b)}, \quad \kappa(b)=\gamma(b)-3 .
\end{aligned}
$$

According to the main philosophy of this method, all functions in the basic deterministic problem (heat conductivity, heat capacity, temperature and its gradient as well as the material density) are expressed similarly to the following finite expansion [7] of a random function $f(\omega)$ :

$f(\omega)=f^{0}(\omega)+\varepsilon \frac{\partial f}{\partial b} \Delta b+\cdots+\frac{1}{n !} \varepsilon^{n} \frac{\partial^{n} f}{\partial b^{n}} \Delta b^{n}$,

where $\varepsilon$ is a given small perturbation (taken usually as equal to 1$), \varepsilon \Delta b$ denotes the first order variation of $b$ from its expected value

$\varepsilon \Delta b=\delta b=\varepsilon\left(b-b^{0}\right)$,

while the $n$th order variation is given as follows:

$\varepsilon^{n} \Delta b^{n}=(\delta b)^{n}=\varepsilon^{n}\left(b-b^{0}\right)^{n}$.

Having solved the equilibrium equations of the considered problem for the state functions and their partial derivatives with respect to the input random variable, one needs to determine the probabilistic moments of the structural response, like temperatures $T(t, b)$. Then, the expected values in the perturbationbased approach are derived as

$$
\begin{aligned}
E[T(t)]= & T^{0}\left(t, b^{0}\right)+\frac{1}{2} \varepsilon^{2} \frac{\partial^{2}(T(t))}{\partial b^{2}} \mu_{2}(b) \\
& +\frac{1}{4 !} \varepsilon^{4} \frac{\partial^{4}(T(t))}{\partial b^{4}} \mu_{4}(b)
\end{aligned}
$$

$$
\begin{aligned}
& +\frac{1}{6 !} \varepsilon^{6} \frac{\partial^{6}(T(t))}{\partial b^{6}} \mu_{6}(b) \\
& +\cdots+\frac{1}{(2 m) !} \varepsilon^{2 m} \frac{\partial^{2 m}(T(t))}{\partial b^{2 m}} \mu_{2 m}(b)
\end{aligned}
$$

for any natural $\mathrm{m}$ with $\mu_{2 m}$ being the central probabilistic moment of $2 m$ th order; let us note that the excluded orders simply equal 0 for the symmetric density functions like the Gaussian one. Usually, according to some previous convergence studies, we may limit this expansion-type approximation to the 10th order [7]. Quite a similar considerations lead to the expressions for higher moments, like the variance, for instance

$$
\begin{aligned}
\operatorname{Var}(T(t)) & \int_{-\infty}^{+\infty}\left(T^{0}(t)+\varepsilon \Delta b \frac{\partial T(t)}{\partial b}+\frac{\varepsilon^{2}}{2}(\Delta b)^{2} \frac{\partial^{2} T(t)}{\partial b^{2}}\right. \\
& +\frac{\varepsilon^{3}}{3 !}(\Delta b)^{3} \frac{\partial^{3} T(t)}{\partial b^{3}}+\frac{\varepsilon^{4}}{4 !}(\Delta b)^{4} \frac{\partial^{4} T(t)}{\partial b^{4}} \\
& \left.+\frac{\varepsilon^{5}}{5 !}(\Delta b)^{5} \frac{\partial^{5} T(t)}{\partial b^{5}}-E[T(t)]\right)^{2} g(b) d b \\
= & \varepsilon^{2} \mu_{2}(b)\left(\frac{\partial T(t)}{\partial b}\right)^{2}+\varepsilon^{4} \mu_{4}(b)\left(\frac{1}{4}\left(\frac{\partial^{2} T(t)}{\partial b^{2}}\right)^{2}\right. \\
& \left.+\frac{2}{3 !} \frac{\partial T(t)}{\partial b} \frac{\partial^{3} T(t)}{\partial b^{3}}\right) \\
& +\varepsilon^{6} \mu_{6}(b)\left(\left(\frac{1}{3 !}\right)^{2}\left(\frac{\partial^{3} T(t)}{\partial b^{3}}\right)^{2}\right. \\
& \left.+\frac{1}{4 !} \frac{\partial^{4} T(t)}{\partial b^{4}} \frac{\partial^{2} T(t)}{\partial b^{2}}+\frac{2}{5 !} \frac{\partial^{5} T(t)}{\partial b^{5}} \frac{\partial T(t)}{\partial b}\right) .
\end{aligned}
$$

The third probabilistic moment may be recovered from this scheme as

$$
\begin{aligned}
\mu_{3}(T(t, b)) \\
=\int_{-\infty}^{+\infty}(T(t, b)-E[T(t, b)])^{3} g(b) d b \\
=\int_{-\infty}^{+\infty}\left(T^{0}(t)+\varepsilon \Delta b \frac{\partial T(t)}{\partial b}+\frac{\varepsilon^{2}}{2}(\Delta b)^{2} \frac{\partial^{2} T(t)}{\partial b^{2}}\right. \\
\quad+\cdots-E[T(t, b)])^{3} g(b) d b \\
=\int_{-\infty}^{+\infty}\left(\varepsilon \Delta b \frac{\partial T(t)}{\partial b}+\frac{\varepsilon^{2}}{2}(\Delta b)^{2} \frac{\partial^{2} T(t)}{\partial b^{2}}\right.
\end{aligned}
$$




$$
\begin{aligned}
& +\cdots)^{3} g(b) d b \\
\cong & \frac{3}{2} \varepsilon^{4} \mu_{4}(b)\left(\frac{\partial T(t)}{\partial b}\right)^{2} \frac{\partial^{2} T(t)}{\partial b^{2}} \\
& +\frac{1}{8} \varepsilon^{6} \mu_{6}(b)\left(\frac{\partial^{2} T(t)}{\partial b^{2}}\right)^{3}
\end{aligned}
$$

using the lowest order approximation; the fourth probabilistic moment computation proceeds from the following formula:

$$
\begin{aligned}
& \mu_{4}(T(t, b)) \\
&=\int_{-\infty}^{+\infty}(T(t, b)-E[T(t, b)])^{4} g(b) d b \\
&=\int_{-\infty}^{+\infty}\left(T^{0}(t)+\varepsilon \Delta b \frac{\partial T(t)}{\partial b}+\frac{\varepsilon^{2}}{2}(\Delta b)^{2} \frac{\partial^{2} T(t)}{\partial b^{2}}\right. \\
&+\cdots-E[T(t, b)])^{4} g(b) d b \\
&= \int_{-\infty}^{+\infty}\left(\varepsilon \Delta b \frac{\partial T(t)}{\partial b}+\frac{\varepsilon^{2}}{2}(\Delta b)^{2} \frac{\partial^{2} T(t)}{\partial b^{2}}\right. \\
&+\cdots)^{4} g(b) d b \\
& \cong \varepsilon^{4} \mu_{4}(b)\left(\frac{\partial T(t)}{\partial b}\right)^{4} \\
&+\frac{3}{2} \varepsilon^{6} \mu_{6}(b)\left(\frac{\partial T(t)}{\partial b} \frac{\partial^{2} T(t)}{\partial b^{2}}\right)^{2} \\
&+\frac{1}{16} \varepsilon^{8} \mu_{8}(b)\left(\frac{\partial^{2} T(t)}{\partial b^{2}}\right)^{4} .
\end{aligned}
$$

As one may suppose, the higher order moments we need to compute the higher order perturbations need to be included into all formulas, so that the complexity of the computational model grows non-proportionally together with the precision and the size of the output information needed.

\subsection{Stochastic ageing processes}

Let us consider a specific class of the non-stationary random process, i.e.

$$
X(\omega, t)=\sum_{i=0}^{n} C_{i}(\omega) t^{i} ; \quad t \in[0, \infty), i \in N
$$

where $C_{i}(\omega)$ are all Gaussian random variables with the specified and bounded first two probabilistic moments. Then, the expected value of this process for the time moment $\tilde{t}$ equals to

$E[X(\omega, \tilde{t})]=\sum_{i=0}^{n} E\left[C_{i}(\omega)\right] \tilde{t}^{i}$.

The variance for this process is defined here as

$\operatorname{Var}(X(\omega, \tilde{t}))=\sum_{i=0}^{n} \operatorname{Var}\left(C_{i}(\omega)\right) \tilde{t}^{2 i}$.

To develop analogous formulas for the higher probabilistic moments we notice that if

$Y(\omega)=a X(\omega), \quad a \in \Re$

then the $k$ th central probabilistic moment is found from its definition as

$$
\begin{aligned}
\mu_{k}(Y(\omega)) & =\int_{-\infty}^{+\infty}(Y(\omega)-E[Y(\omega)])^{k} p(\omega) d \omega \\
& =\int_{-\infty}^{+\infty}(a X(\omega)-a E[X(\omega)])^{k} p(\omega) d \omega \\
& =a^{k} \mu_{k}(X) .
\end{aligned}
$$

Turning back to the initial process one obtains

$$
\left\{\begin{array}{l}
\mu_{3}(X(\omega, \tilde{t}))=\sum_{i=0}^{n} \mu_{3}\left(C_{i}(\omega)\right) \tilde{t}^{3 i}, \\
\mu_{4}(X(\omega, \tilde{t}))=\sum_{i=0}^{n} \mu_{4}\left(C_{i}(\omega)\right) \tilde{t}^{4 i}
\end{array}\right.
$$

and the general expression yields here

$$
\mu_{k}(X(\omega, \tilde{t}))=\sum_{i=0}^{n} \mu_{k}\left(C_{i}(\omega)\right) \tilde{t}^{k i}, \quad k \in N .
$$

The basic coefficients as variation, asymmetry and flatness are simply determined as

$$
\begin{aligned}
& \alpha(X(\omega, \tilde{t}))=\frac{\sqrt{\sum_{i=0}^{n} \operatorname{Var}\left(C_{i}(\omega)\right) \tilde{t}^{2 i}}}{\sum_{i=0}^{n} E\left[C_{i}(\omega)\right] \tilde{t}^{i}}, \\
& \beta(X(\omega, \tilde{t}))=\frac{\sum_{i=0}^{n} \mu_{3}\left(C_{i}(\omega)\right) \tilde{t}^{3 i}}{\left(\sum_{i=0}^{n} \operatorname{Var}\left(C_{i}(\omega)\right) \tilde{t}^{2 i}\right)^{\frac{3}{2}}}, \\
& \gamma(X(\omega, \tilde{t}))=\frac{\sum_{i=0}^{n} \mu_{4}\left(C_{i}(\omega)\right) \tilde{t}^{4 i}}{\left(\sum_{i=0}^{n} \operatorname{Var}\left(C_{i}(\omega)\right) \tilde{t}^{2 i}\right)^{2}} .
\end{aligned}
$$


Since $C_{i}(\omega)$ are all Gaussian, then only half of those moments have non-zero values, i.e.

$\mu_{2 l}(X(\omega, \tilde{t}))=\sum_{i=0}^{n} \mu_{2 l}\left(C_{i}(\omega)\right) \tilde{t}^{2 l i}, \quad l \in N$.

It can be relatively easy to prove that the random process given by (11) is non-stationary being at the same continuous in time and having continuous realizations.

\section{Variational formulation of the thermo-piezoelectric problem}

Let us consider a free energy of the thermo-piezoelectric continuum consisting of thermal, electric and mechanical fields contributions as [13, 14]

$$
\begin{aligned}
W\left(\varepsilon_{i j}, E_{i}, \Theta\right)= & \frac{1}{2} C_{i j k l} \varepsilon_{i j} \varepsilon_{k l}-e_{i j k} E_{i} \varepsilon_{j k} \\
& -\frac{1}{2} b_{i j} E_{i} E_{j}-k_{i j} \theta \varepsilon_{i j}-d_{i} E_{i} \theta \\
& -\frac{1}{2} \alpha_{T} \theta^{2} .
\end{aligned}
$$

In the above equation (20): $\varepsilon_{i j}$ denotes the components of the overall strain tensor, $E_{i}$ - the components of the electric field vector, $\theta$-actual temperature increment from the reference temperature $\theta_{0}$ defining the state with no initial stresses nor strains. The fourth and third order tensors $C_{i j k l}$ and $e_{i j k}$ contain the elastic and piezoelectric constants, while $b_{i j}$ stands for the dielectric permittivity; the tensors $k_{i j}$ and $d_{i}$ represent thermo-mechanical and thermo-piezoelectric coupling constants, where $\alpha_{T}=\frac{c}{\theta_{0}}$ relates the heat capacity to the reference temperature. Additionally, the dissipation function $F$ is considered here as

$$
F\left(e_{i}\right)=\frac{1}{2} \lambda_{i j} e_{i} e_{j},
$$

where $\lambda_{i j}$ means the heat conductivity tensor and $e_{i}$ is the thermal field vector. As one knows, the constitutive equations are derived from (20), whereas the Fourier's law is a consequence of the dissipation function. There holds

$\sigma_{i j}=\frac{\partial W\left(\varepsilon_{i j}, E_{i}, \Theta\right)}{\partial \varepsilon_{i j}}=C_{i j k l} \varepsilon_{k l}-e_{i j k} E_{k}-k_{i j} \theta$,
$D_{i}=-\frac{\partial W\left(\varepsilon_{i j}, E_{i}, \Theta\right)}{\partial E_{i}}=e_{i j k} \varepsilon_{j k}+b_{i j} E_{j}+d_{i} \theta$,

$S=-\frac{\partial W\left(\varepsilon_{i j}, E_{i}, \Theta\right)}{\partial \theta}=k_{i j} \varepsilon_{i j}+d_{i} E_{i}+\alpha_{T} \theta$,

$q_{i}=\frac{\partial F\left(e_{i}\right)}{\partial e_{i}}=\lambda_{i j} e_{j}$

The stress tensor components $\sigma_{i j}$, the components of the electric displacement $D_{i}$, the entropy $S$ and the components of the heat flux vector $q_{i}$ are determined uniquely from the above equations. The following assumptions accompany this solution and there are:

(1) small displacements limitation, where

$$
\varepsilon_{k l}=\frac{1}{2}\left(\frac{\partial u_{k}}{\partial x_{l}}+\frac{\partial u_{l}}{\partial x_{k}}\right),
$$

(2) linear piezoelectricity

$$
E_{i}=-\Phi_{, i},
$$

where $\Phi$ denotes the scalar potential function,

(3) the following description of the thermal field vector:

$$
e_{i}=-\theta_{, i}
$$

Finally, the Hamilton's principle is used

$\delta \int_{t_{1}}^{t_{2}}(L+W) d t=0$

where the $L$ and $W$ include all thermal, electric and mechanical contributions with all variations (of displacements, temperatures etc.) vanishing at the beginning $t_{1}$ and at the end $t_{2}$ of the considered time period; the Lagrangian $L$ is defined here as follows:

$L=\int_{\Omega}\left(K+F-W-S \theta-S \theta_{0} \dot{\theta}\right) d \Omega$

where

$K=\frac{1}{2} \rho \dot{u}_{i} \dot{u}_{i}$

is adequate to the kinetic energy, while the remaining components become

$W=\frac{1}{2} \sigma_{i j} \varepsilon_{i j}-\frac{1}{2} D_{i} E_{i}-\frac{1}{2} S \theta$, 
$F=\frac{1}{2} q_{i} e_{i}$.

Determining all variations with respect to displacements, temperature and electric potential one can obtain the following set of the variational equations having unique solution [11]:

$$
\begin{aligned}
\delta_{u}(L+W)= & -\int_{t_{1}}^{t_{2}} \int_{\Omega}\left(\rho \ddot{u}_{i} \delta u_{i}+\sigma_{i j} \delta \varepsilon_{i j}\right) d \Omega d t \\
& +\int_{t_{1}}^{t_{2}} \int_{\partial \Omega} t_{i} \delta u_{i} d(\partial \Omega) d t=0, \\
\delta_{\Phi}(L+W)= & -\int_{t_{1}}^{t_{2}} \int_{\Omega} D_{i} \delta \Phi_{, i} d \Omega d t \\
& +\int_{t_{1}}^{t_{2}} \int_{\partial \Omega} q_{e} \delta \Phi d(\partial \Omega) d t=0, \\
\delta_{\theta}(L+W)= & \int_{t_{1}}^{t_{2}} \int_{\Omega}\left(-q_{i} \delta \theta_{, i}+\dot{S} \theta_{0} \delta \theta\right) d \Omega d t \\
& +\int_{t_{1}}^{t_{2}} \int_{\partial \Omega} q_{S} \delta \theta d(\partial \Omega) d t=0 .
\end{aligned}
$$

Those equations are valid for some continuum $\Omega$ with its external boundary $\partial \Omega$, where the boundary conditions expressed by $t_{i}, q_{e}$ and $q_{S}$ representing the boundary forces, the charge density and the boundary heat fluxes, are uniquely defined.

The variational equations (34)-(36) are now reformulated in the context of the stochastic perturbation technique and, for the needs of computational implementation, they are presented in the recursive form. It starts traditionally from the zeroth order equations, being in fact, almost the same like those given above. There holds

$$
\begin{aligned}
& \int_{t_{1}}^{t_{2}} \int_{\Omega}\left(\rho^{0} \ddot{u}_{i}^{0} \delta u_{i}+\sigma_{i j}^{0} \delta \varepsilon_{i j}\right) d \Omega d t \\
& =\int_{t_{1}}^{t_{2}} \int_{\partial \Omega} t_{i}^{0} \delta u_{i} d(\partial \Omega) d t \\
& \int_{t_{1}}^{t_{2}} \int_{\Omega} D_{i}^{0} \delta \Phi_{, i} d \Omega d t=\int_{t_{1}}^{t_{2}} \int_{\partial \Omega} q_{e}^{0} \delta \Phi d(\partial \Omega) d t \\
& \int_{t_{1}}^{t_{2}} \int_{\Omega}\left(q_{i}^{0} \delta \theta_{, i}-\dot{S}^{0} \theta_{0}^{0} \delta \theta\right) d \Omega d t \\
& =\int_{t_{1}}^{t_{2}} \int_{\partial \Omega} q_{S}^{0} \delta \theta d(\partial \Omega) d t
\end{aligned}
$$

Having solved zeroth order quantities, the higher order responses need to be computed. Therefore, the general $n$th order variational equations are introduced as

$$
\begin{aligned}
& \int_{t_{1}}^{t_{2}} \int_{\Omega}\left(\sum_{k=0}^{n}\left(\begin{array}{l}
n \\
k
\end{array}\right) \rho^{(k)} \ddot{u}_{i}^{(n-k)} \delta u_{i}+\sigma_{i j}^{(n)} \delta \varepsilon_{i j}\right) d \Omega d t \\
& =\int_{t_{1}}^{t_{2}} \int_{\partial \Omega} t_{i}^{(n)} \delta u_{i} d(\partial \Omega) d t \\
& \int_{t_{1}}^{t_{2}} \int_{\Omega} D_{i}^{(n)} \delta \Phi_{, i} d \Omega d t=\int_{t_{1}}^{t_{2}} \int_{\partial \Omega} q_{e}^{(n)} \delta \Phi d(\partial \Omega) d t \\
& \int_{t_{1}}^{t_{2}} \int_{\Omega}\left(q_{i}^{(n)} \delta \theta_{, i}-\sum_{k=0}^{n}\left(\begin{array}{l}
n \\
k
\end{array}\right) \dot{S}^{(k)} \theta_{0}^{(n-k)} \delta \theta\right) d \Omega d t \\
& =\int_{t_{1}}^{t_{2}} \int_{\partial \Omega} q_{S}^{(n)} \delta \theta d(\partial \Omega) d t
\end{aligned}
$$

where $\sigma_{i j}^{(n)}$ is equivalent to the $n$th order partial derivative of the stress tensor with respect to the input random variable etc. The remaining part of the solution proceeds relatively easily_zeroth order solutions are inserted into the first order versions of (40)-(42), where the first order solutions are extracted from to be included into the second order equations etc. A choice of the highest order stochastic expansion strongly depends on the desired accuracy of the computational process and needs to be the subject of the separate analysis. As it is demonstrated, the straightforward differentiation technique applied to the Hamilton's principle may be replaced with some alternative techniques to determine the partial derivatives of the structural response with respect to some random variables.

Finally, let us mention that some analytical solutions to such coupled problems (even with magnetic fields contribution) are available [5, 16], but they are valid for specific domains or components distribution in space, so that it is impossible to provide any general probabilistic methodology on their basis; one may use in those cases the Monte-Carlo simulation only to calculate analytically the statistical parameters for the structural response.

\section{The perturbation-based stochastic finite element method formulation}

Let us introduce now the additional Stochastic Finite Element Method discretization of the problem given 
by (37)-(42). It starts from the traditional vectorization of the displacement field $\mathbf{u}$, the electric potential $\boldsymbol{\Phi}$ and the temperatures $\boldsymbol{\theta}$. It is completed using their nodal values $\mathbf{q}, \Psi, T$ and the shape functions $\varphi_{\mathbf{q}}, \varphi_{\Psi}$ as well as $\varphi_{\mathbf{T}}$ as follows:

$\mathbf{u}=\varphi_{\mathbf{q}} \mathbf{q}, \quad \Phi=\varphi_{\Psi} \Psi, \quad \boldsymbol{\theta}=\varphi_{T} \mathbf{T}$.

So that, the strain tensor $\boldsymbol{\varepsilon}$, the electric field $\mathbf{E}$ and the thermal field $\mathbf{e}$ (cf. (26)-(28)) are related to the vectorized state functions as

$\boldsymbol{\varepsilon}=\nabla \varphi_{\mathbf{q}} \mathbf{q}=\mathbf{B}_{\mathbf{q}} \mathbf{q}$,

$\mathbf{E}=-\nabla \varphi_{\Psi} \Psi=\mathbf{B}_{\Psi} \Psi$,

$\mathbf{e}=-\nabla \varphi_{T} \mathbf{T}=\mathbf{B}_{\mathbf{T}} \mathbf{T}$.

The constitutive equations (22)-(25) become in this manner equal to

$\sigma=\mathbf{C} \varepsilon-\mathbf{P E}-\mathbf{k T}$,

$\mathbf{D}=\mathbf{P}^{\mathbf{T}} \boldsymbol{\varepsilon}+\mathbf{B E}+\mathbf{d T}$,

$\mathbf{S}=\mathbf{k}^{\mathbf{T}} \varepsilon+\mathbf{d}^{\mathbf{T}} \mathbf{E}+\boldsymbol{\alpha}_{\mathbf{T}} \mathbf{T}$,

$\mathbf{q}=\lambda \mathbf{e}$,

where $\sigma, \mathbf{D}$ are the stress tensor components and the electric displacement vector, and matrices $\mathbf{C}, \mathbf{B}, \mathbf{P}, \mathbf{k}$, $\mathbf{d}$ and $\lambda$ denote the elasticity, dielectric permittivity, piezoelectric constant, thermo-mechanical coupling, thermo-piezoelectric coupling and thermal conductivity tensors. Then, we introduce this discretization into the variational equations (40)-(42); therefore, the fundamental set of equilibrium equations for the thermopiezoelectric problem becomes

$$
\begin{aligned}
\left(\begin{array}{ccc}
\mathbf{M}_{\mathbf{u u}} & \mathbf{0} & \mathbf{0} \\
\text { symm. } & \mathbf{0} & \mathbf{0}
\end{array}\right)\left(\begin{array}{c}
\ddot{\mathbf{q}} \\
\ddot{\Psi} \\
\ddot{\mathbf{T}}
\end{array}\right) \\
+\left(\begin{array}{ccc}
\mathbf{0} & \mathbf{0} & \mathbf{0} \\
\mathbf{0} & \mathbf{0} & \mathbf{0} \\
\mathbf{C}_{\mathbf{T u}} & \mathbf{C}_{\mathbf{T} \Psi} & \mathbf{C}_{\mathbf{T T}}
\end{array}\right)\left(\begin{array}{c}
\dot{\mathbf{q}} \\
\dot{\Psi} \\
\dot{\mathbf{T}}
\end{array}\right) \\
+\left(\begin{array}{ccc}
\mathbf{K}_{\mathbf{u u}} & \mathbf{K}_{\mathbf{u} \Psi} & \mathbf{K}_{\mathbf{u T}} \\
\mathbf{K}_{\Psi \mathbf{u}} & \mathbf{K}_{\Psi \Psi} & \mathbf{K}_{\Psi \mathbf{T}} \\
\mathbf{0} & \mathbf{0} & \mathbf{K}_{\mathbf{T T}}
\end{array}\right)\left(\begin{array}{c}
\mathbf{q} \\
\Psi \\
\mathbf{T}
\end{array}\right) \\
=\left(\begin{array}{c}
\mathbf{F}_{\mathbf{u}} \\
\mathbf{F}_{\Psi} \\
\mathbf{F}_{\mathbf{T}}
\end{array}\right),
\end{aligned}
$$

where $\mathbf{M}_{\mathbf{u u}}$ is the mass matrix, $\mathbf{C}_{\mathbf{T u}}, \mathbf{C}_{\mathbf{T} \boldsymbol{\Phi}}$ and $\mathbf{C}_{\mathbf{T T}}$ are the damping matrices resulting from the thermomechanical, thermo-electric couplings and thermal field, respectively. The matrices $\mathbf{K}_{\mathbf{u} \Psi}, \mathbf{K}_{\mathbf{\Psi} \mathbf{u}}, \mathbf{K}_{\mathbf{u T}}$, $\mathbf{K}_{\Psi \mathbf{T}}, \mathbf{K}_{\mathrm{uu}}, \mathbf{K}_{\Psi \Psi}, \mathbf{K}_{\mathrm{TT}}$ are the stiffness matrices related to the piezoelectric-mechanical (first two), thermo-mechanical, thermo-electric couplings as well as the stiffnesses adjacent to the mechanical, electric and thermal fields, correspondingly. Finally, the RHS vectors $\mathbf{F}_{\mathbf{u}}, \mathbf{F}_{\boldsymbol{\Psi}}$ as well as $\mathbf{F}_{\mathbf{T}}$ represent the mechanical, electric and thermal boundary conditions. Their definitions are given as follows:

$$
\begin{aligned}
& M_{u u}=\int_{\Omega} N_{u}^{T} \rho N_{u} d \Omega, \\
& C_{T u}=-\int_{\Omega} T_{0} N_{T}^{T} \lambda^{T} B_{u} d \Omega, \\
& C_{T \Psi}=-\int_{\Omega} T_{0} N_{T}^{T} d^{T} B_{\Psi} d \Omega, \\
& C_{T T}=-\int_{\Omega} T_{0} N_{T}^{T} \alpha_{T} N_{T} d \Omega, \\
& K_{u u}=\int_{\Omega} B_{u}^{T} Q B_{u} d \Omega, \\
& K_{u \Psi}=-\int_{\Omega} B_{u}^{T} P B_{\Psi} d \Omega, \\
& K_{u T}=-\int_{\Omega} B_{u}^{T} \lambda N_{T} d \Omega, \\
& K_{\Psi u}=-\int_{\Omega} B_{\Psi}^{T} P^{T} B_{u} d \Omega, \\
& K_{\Psi \Psi}=\int_{\Omega} B_{\Psi}^{T} B B_{\Psi} d \Omega, \\
& K_{\Psi T}=-\int_{\Omega} B_{\Psi}^{T} d N_{T} d \Omega, \\
& K_{T T}=-\int_{\Omega} B_{T}^{T} \kappa B_{T} d \Omega .
\end{aligned}
$$

Let us also note that

$$
\begin{aligned}
& K_{\Psi u}=K_{u \Psi}^{T}, \quad C_{T u}=T_{0} K_{u T}^{T}, \\
& C_{T \Psi}=T_{0} K_{\Psi T}^{T} .
\end{aligned}
$$

On this basis we may write the zeroth and the $n$th order stochastic equations systems corresponding to the 
deterministic system given by (51) as follows:

$$
\begin{aligned}
& \left(\begin{array}{ccc}
\mathbf{M}_{\mathbf{u u}}^{(0)} & \mathbf{0} & \mathbf{0} \\
& \mathbf{0} & \mathbf{0} \\
\text { symm. } & & \mathbf{0}
\end{array}\right)\left(\begin{array}{c}
\ddot{\mathbf{q}}^{(0)} \\
\ddot{\boldsymbol{\Psi}}^{(0)} \\
\ddot{\mathbf{T}}^{(0)}
\end{array}\right) \\
& +\left(\begin{array}{ccc}
\mathbf{0} & \mathbf{0} & \mathbf{0} \\
\mathbf{0} & \mathbf{0} & \mathbf{0} \\
\mathbf{C}_{\mathbf{T u}}^{(0)} & \mathbf{C}_{\mathbf{T} \boldsymbol{\Psi}}^{(0)} & \mathbf{C}_{\mathbf{T T}}^{(0)}
\end{array}\right)\left(\begin{array}{c}
\dot{\mathbf{q}}^{(0)} \\
\dot{\boldsymbol{\Psi}}^{(0)} \\
\dot{\mathbf{T}}^{(0)}
\end{array}\right) \\
& +\left(\begin{array}{ccc}
\mathbf{K}_{\mathbf{u u}}^{(0)} & \mathbf{K}_{\mathbf{u} \boldsymbol{\Psi}}^{(0)} & \mathbf{K}_{\mathbf{u T}}^{(0)} \\
\mathbf{K}_{\mathbf{\Psi u}}^{(0)} & \mathbf{K}_{\boldsymbol{\Psi} \boldsymbol{\Psi}}^{(0)} & \mathbf{K}_{\mathbf{\Psi} \mathbf{T}}^{(0)} \\
\mathbf{0} & \mathbf{0} & \mathbf{K}_{\mathbf{T T}}^{(0)}
\end{array}\right)\left(\begin{array}{c}
\mathbf{q}^{(0)} \\
\boldsymbol{\Psi}^{(0)} \\
\mathbf{T}^{(0)}
\end{array}\right) \\
& =\left(\begin{array}{c}
\mathbf{F}_{\mathbf{u}}^{(0)} \\
\mathbf{F}_{\Psi}^{(0)} \\
\mathbf{F}_{\mathbf{T}}^{(0)}
\end{array}\right)
\end{aligned}
$$

and

$$
\begin{aligned}
\sum_{k=0}^{n}\left(\begin{array}{l}
n \\
k
\end{array}\right)\left(\begin{array}{ccc}
\mathbf{M}_{\mathbf{u u}}^{(k)} & \mathbf{0} & \mathbf{0} \\
& \mathbf{0} & \mathbf{0} \\
\text { symm. } & & \mathbf{0}
\end{array}\right)\left(\begin{array}{c}
\ddot{\mathbf{q}}^{(n-k)} \\
\ddot{\mathbf{\Psi}}^{(n-k)} \\
\ddot{\mathbf{T}}^{(n-k)}
\end{array}\right) \\
+\sum_{k=0}^{n}\left(\begin{array}{l}
n \\
k
\end{array}\right)\left(\begin{array}{ccc}
\mathbf{0} & \mathbf{0} & \mathbf{0} \\
\mathbf{0} & \mathbf{0} & \mathbf{0} \\
\mathbf{C}_{\mathbf{T u}}^{(k)} & \mathbf{C}_{\mathbf{T \Psi}}^{(k)} & \mathbf{C}_{\mathbf{T T}}^{(k)}
\end{array}\right)\left(\begin{array}{c}
\dot{\mathbf{q}}^{(n-k)} \\
\dot{\boldsymbol{\Psi}}^{(n-k)} \\
\dot{\mathbf{T}}^{(n-k)}
\end{array}\right) \\
+\sum_{k=0}^{n}\left(\begin{array}{l}
n \\
k
\end{array}\right)\left(\begin{array}{ccc}
\mathbf{K}_{\mathbf{u u}}^{(k)} & \mathbf{K}_{\mathbf{u} \Psi}^{(k)} & \mathbf{K}_{\mathbf{u T}}^{(k)} \\
\mathbf{K}_{\mathbf{\Psi} \mathbf{u}}^{(k)} & \mathbf{K}_{\mathbf{\Psi} \Psi}^{(k)} & \mathbf{K}_{\mathbf{\Psi} \mathbf{T}}^{(k)} \\
\mathbf{0} & \mathbf{0} & \mathbf{K}_{\mathbf{T T}}^{(k)}
\end{array}\right)\left(\begin{array}{c}
\mathbf{q}^{(n-k)} \\
\boldsymbol{\Psi}^{(n-k)} \\
\mathbf{T}^{(n-k)}
\end{array}\right) \\
=\left(\begin{array}{l}
\mathbf{F}_{\mathbf{u}}^{(n)} \\
\mathbf{F}_{\mathbf{\Psi}}^{(n)} \\
\mathbf{F}_{\mathbf{T}}^{(n)}
\end{array}\right)
\end{aligned}
$$

which include all the variational statements formulated in (40)-(42). Let us note that contrary to the initial equation (58) most of the submatrices in (59) equal 0 , especially for a single random variable, since this variable appears effectively in the few of them, frequently in the lower order polynomial form.

\section{Thermopiezoelectric coupling in a straight beam}

Let us consider the equation of motion of an elastic beam as

$\rho \frac{\partial^{2} u}{\partial t^{2}}=\frac{\partial \sigma}{\partial x}$ where the normal stresses in the beam are defined as

$\sigma=\left(E+\frac{e^{2}}{b}\right) \varepsilon-\frac{e}{b} D-\alpha E \theta$.

A substitution of the stresses into the equation of motion yields

$\rho \frac{\partial^{2} u}{\partial t^{2}}=\left(E+\frac{e^{2}}{b}\right) \frac{\partial^{2} u}{\partial x^{2}}-\alpha E \frac{\partial \theta}{\partial x}$,

while the heat transfer equation with thermo-piezoelectric coupling takes the following form [10]:

$k \frac{\partial^{2} \theta}{\partial x^{2}}=E \alpha \theta_{0} \frac{\partial}{\partial t}\left(\frac{\partial u}{\partial x}\right)+c \frac{\partial \theta}{\partial t}$.

The complex pulsation is obtained by the following harmonic representation of the additional solutions:

$u=U_{0} \exp (i \omega t) ; \quad \theta=\theta_{0} \exp (i \omega t)$,

where the first mechanical and thermal modes are given by

$U_{0}=A \sin \left(\frac{\pi x}{l}\right) ; \quad \theta_{0}=B \cos \left(\frac{\pi x}{l}\right)$,

where $A$ and $B$ depend on each other. Substituting those representations into the relation (62) one obtains

$B=A\left(\frac{\pi}{l} \frac{1}{\alpha}\left(1+\frac{e^{2}}{E b}\right)-\omega^{2} \frac{l}{\pi} \frac{\rho}{E \alpha}\right)$.

Therefore, the thermo-piezoelectric pulsation $\omega$ of this beam is the real eigenvalue fulfilling the following equation:

$$
\begin{aligned}
- & \frac{\rho}{E}\left(\frac{l}{\pi}\right)^{2} i \omega^{3}-\frac{\rho}{E} \frac{k}{c} \omega^{2}+\left(1+\frac{e^{2}}{E b}+\frac{\theta_{0} \alpha^{2} E}{c}\right) i \omega \\
+ & \frac{k}{c}\left(\frac{\pi}{l}\right)^{2}\left(1+\frac{e^{2}}{E b}\right)=0
\end{aligned}
$$

\section{Computational analysis}

Computational analysis obeys here deterministic sensitivity analysis with respect to the beam length and piezoelectric constant (Sect. 6.1), probabilistic analysis with random beam length (Sect. 6.2) and stochastic computations, where once more the beam length is subjected to the ageing process (Sect. 6.3). This 
choice is driven by the results of previous computational analyses, where the so-called quality factor was presented as a function of this particular parameter [2]. All the computational experiments are devoted to the silicon beam having in a deterministic case the following properties [10]:

$E=1.58 \times 10^{11} \frac{\mathrm{N}}{\mathrm{m}^{2}}, \quad \rho=2300 \frac{\mathrm{kg}}{\mathrm{m}^{3}}$,

$e=0.1711 \frac{\mathrm{C}}{\mathrm{m}^{2}}, \quad b=3.992 \times 10^{-11} \frac{\mathrm{F}}{\mathrm{m}^{2}}$,

$c=711 \frac{\mathrm{J}}{\mathrm{kg} \mathrm{K}}, \quad \alpha_{T}=2.5 \times 10^{-6} \frac{1}{\mathrm{~K}}$,

$k=170 \frac{\mathrm{W}}{\mathrm{mK}}, \quad T_{0}=298 \mathrm{~K}$ and $l=3.9 \times 10^{-8} \mathrm{~m}$,

which lead to the single real solution to (67), not available in the analytical form in symbolic environment of the system MAPLE. Once this analytical solution appears to be available one may apply the fundamental definitions of the probability theory to determine exactly the basic probabilistic characteristics of thermopulsation frequency. Further, it is apparent that the silicon beam length is convenient to the nanoscale rather than to the micro- or macroscale adjacent to the equations provided in Sect. 3, however the results contained in [10] show the very good agreement with the other theories in that area. On the other hand, an extension of the approach proposed towards the stochastic nanomechanics would be very challenging and interesting - by the use of Lennard-Jones potentials apparatus, for instance.

Numerical approach presented here is based on polynomial approximation of partial derivatives of the structural response with respect to the input design (random) variable included in (4) through the several of solutions to the input deterministic problem. They are completed for the set of different values of this variable chosen uniformly around its mean valuethe polynomial interrelation between the final thermopulsation and some input parameters is provided numerically using 11-points tests. As one may recognize the approximants are smooth, without local oscillations, and match perfectly everywhere the trial points set, so that all partial derivatives determination of the increasing order proceeds effectively.

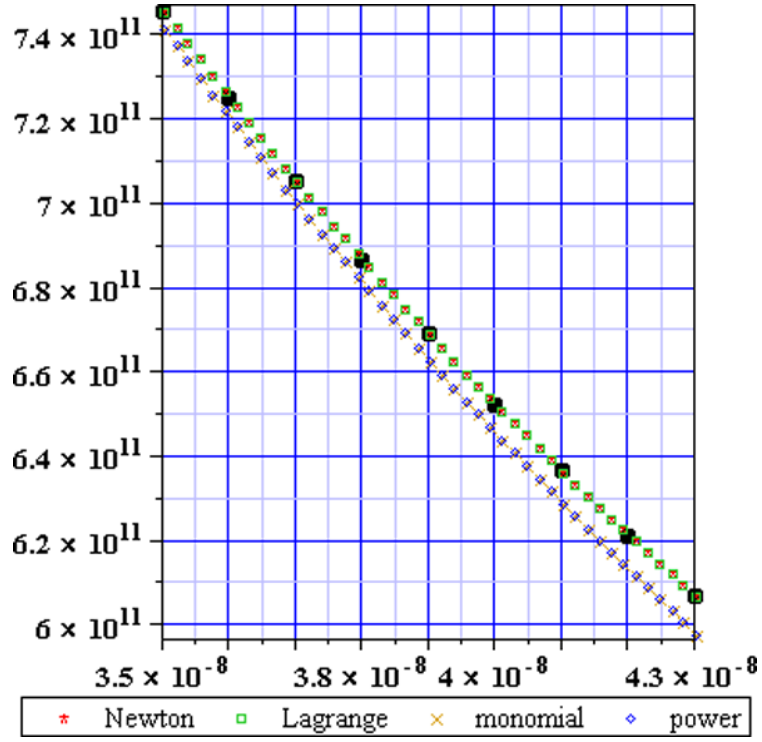

Fig. 1 Thermo-pulsation variations with respect to input specimen length

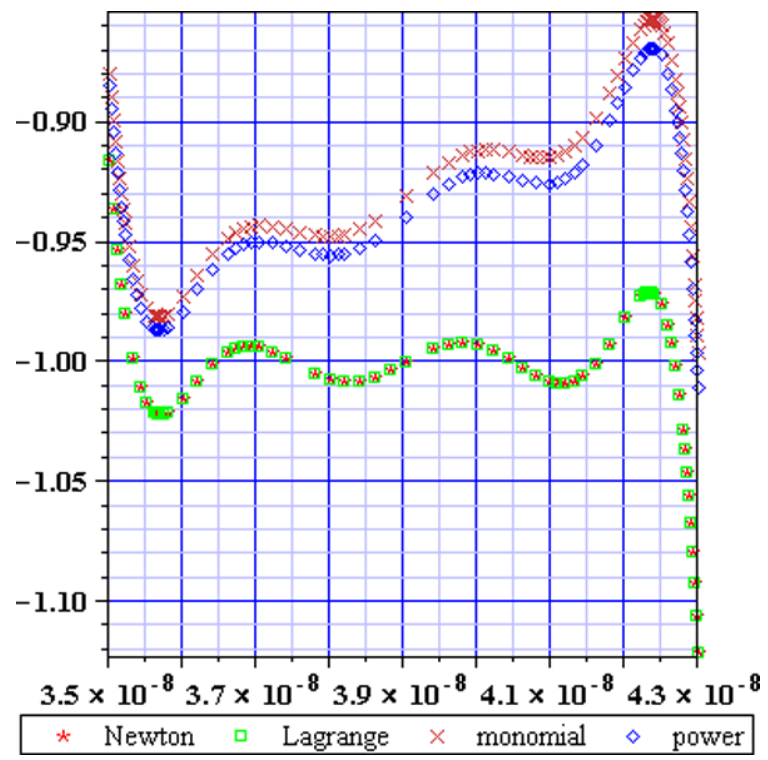

Fig. 2 First order sensitivity of thermo-pulsation with respect to input specimen length

\subsection{Deterministic sensitivity analysis}

The results of numerical design sensitivity analysis are presented in Figs. 1-6 for two independent design variables - the beam length (Figs. 1-3) and the piezoelectric constant (Figs. 4-6). Each time we present the set of various approximations for the interrelation 


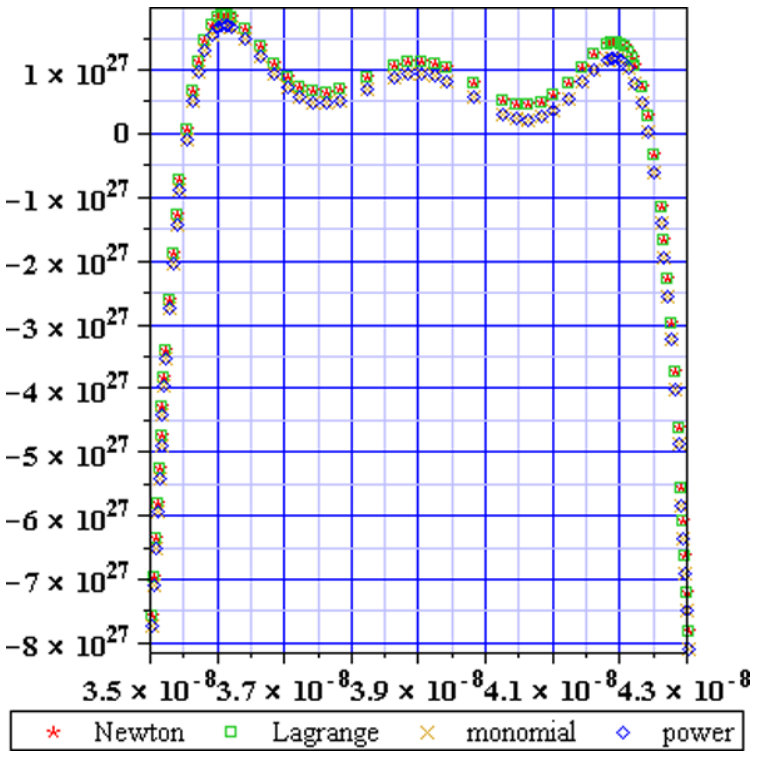

Fig. 3 Second order sensitivity of thermo-pulsation with respect to input specimen length

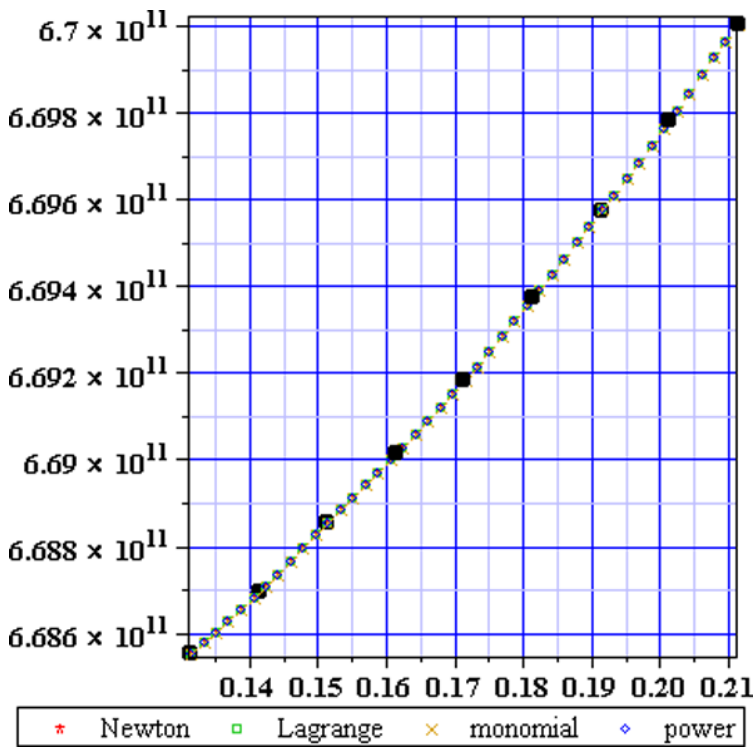

Fig. 4 Thermo-pulsation variations with respect to the piezoelectric constant

between the thermo-pulsation and the given variable within some neighborhood of its mean value and, further, first and second order sensitivities of this thermopulsation to this parameter within the same domain. Each time we explore the usage of Newton, Lagrange, monomial and power approximations of this response function and their influence on the final values of the

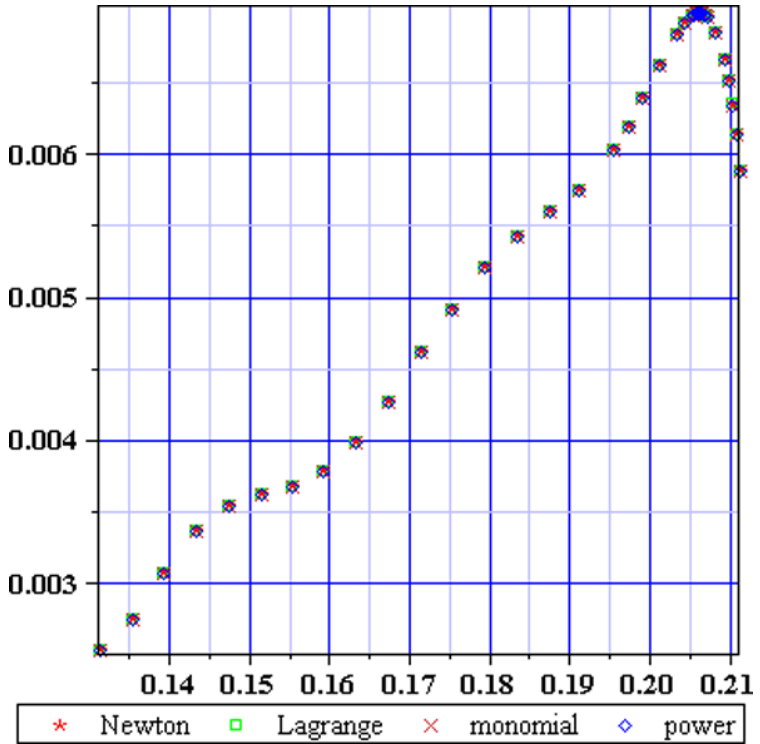

Fig. 5 First order sensitivity of thermo-pulsation with respect to the piezoelectric constant



* Newton $\square$ Lagrange $\times$ monomial $\diamond$ power

Fig. 6 Second order sensitivity of thermo-pulsation with respect to the piezoelectric constant

sensitivity gradients. As it is documented by Fig. 1 the proposed approximators not always match perfectly the set of trial points (shown here by black dots)—only Newton and Lagrange methods are efficient in this context. Following this result the first and the second order sensitivities (cf. Figs. 2 and 3) computed according to the monomial and power methods return slightly 
different results-a difference at the mean value is about $5 \%$ for the first derivative and the few orders smaller for the second order derivative. We notice also that all the sensitivities have clear oscillatory character around the mean value of the beam length; fortunately since the initial purpose was to determine those sensitivities for the design parameter mean value only, those variations at the upper and lower bound for the computational domain are of the secondary importance. Figure 3 demonstrates that the second order sensitivities computed at those bounds have even opposite sign to the sensitivity adjacent to the mean value. Physically we found that an increase of the beam length decreases the final thermo-pulsation value and that their algebraic interrelation is convex.

The sets of Figs. 4-6 equivalent to the piezoelectric constant as the design parameter show that when all the methods return the approximation matching perfectly the set of initial problem iterative solutions (Fig. 4), then the remaining results agree with each other almost perfectly. Even in this case the sensitivities computations result in some oscillations increasing together with the distance from the mean value. Now, both sensitivity gradients are positive, which means that the increase of the piezoelectric constant increases the thermo-pulsation being studied and that those two parameters interrelation is convex; let us note that this interrelation is almost linear within the assumed computational domain.

\subsection{Probabilistic analysis}

It is assumed now that the beam length is the Gaussian random variable having the expected value equal to the main value taken initially; the design parameter of this study is the coefficient of variation for this variable. Computational results obtained in the system MAPLE thanks to the Response Function Method [7] using the polynomial approximation obtained before (Fig. 1) and the additional Taylor expansion application are presented below as the expected values (Fig. 7), the standard deviations (Fig. 8), the coefficients of variation (Fig. 9), the third (Fig. 10) and the fourth central probabilistic moments (Fig. 11) of the beam pulsation (the real eigenvalue) returned as the solution to (67); they are presented as the functions of the coefficients of variation of the beam length for $\alpha(l) \in[0.0,0.25]$. They are all contrasted with the Monte-Carlo simulation for the total number of random trials $N=50.000$,

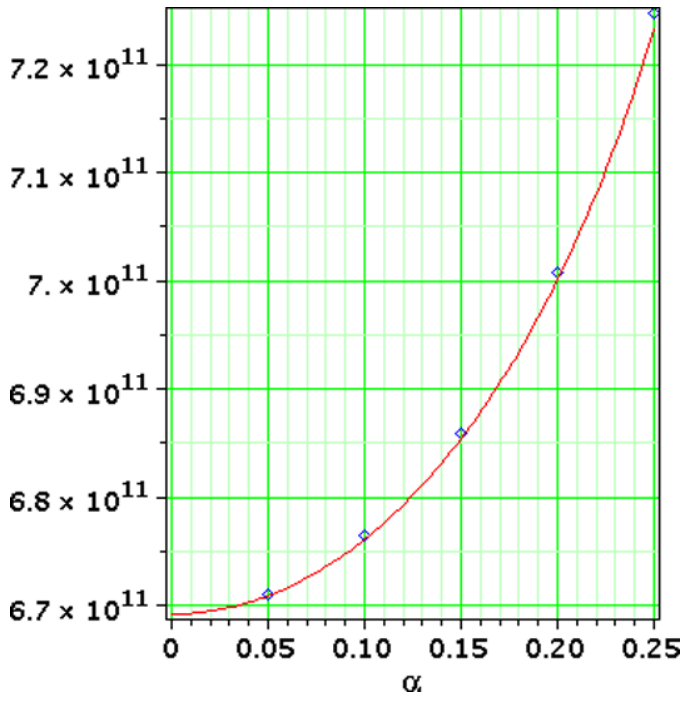

Fig. 7 The expected values of the beam pulsation frequency

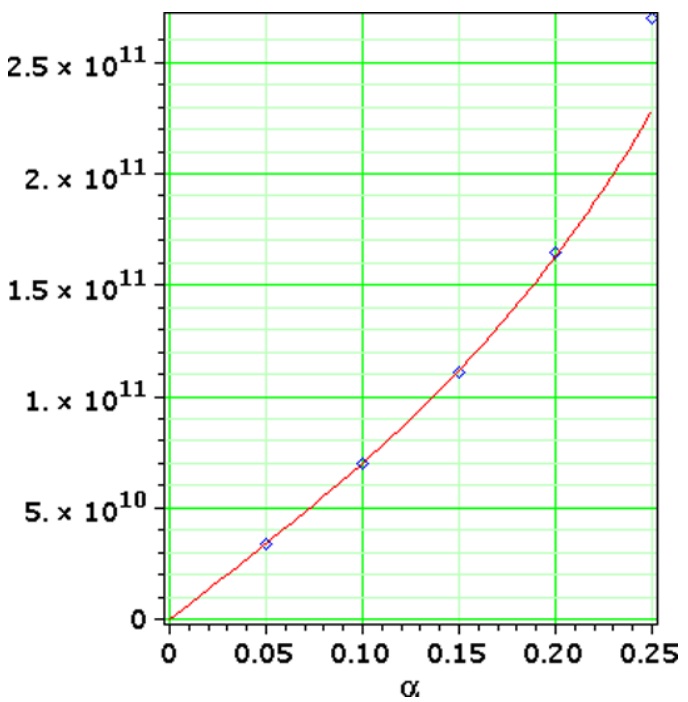

Fig. 8 The standard deviations of the beam pulsation frequency

also carried out in MAPLE, v. 13. Many of the computational experiments provided before shows that this number of simulations guarantees satisfactory probabilistic convergence of up to the fourth central probabilistic moments being studied now. Contrary to the very fast perturbation-based analysis (less than $60 \mathrm{sec}-$ onds), it takes more than 18.000 seconds for each value of $\alpha(l)$. Those computations were provided in the discrete mode with respect to this coefficient each time taken from the set $[0.05,0.10,0.15,0.20,0.25]$. So that the perturbation-based results are marked with 


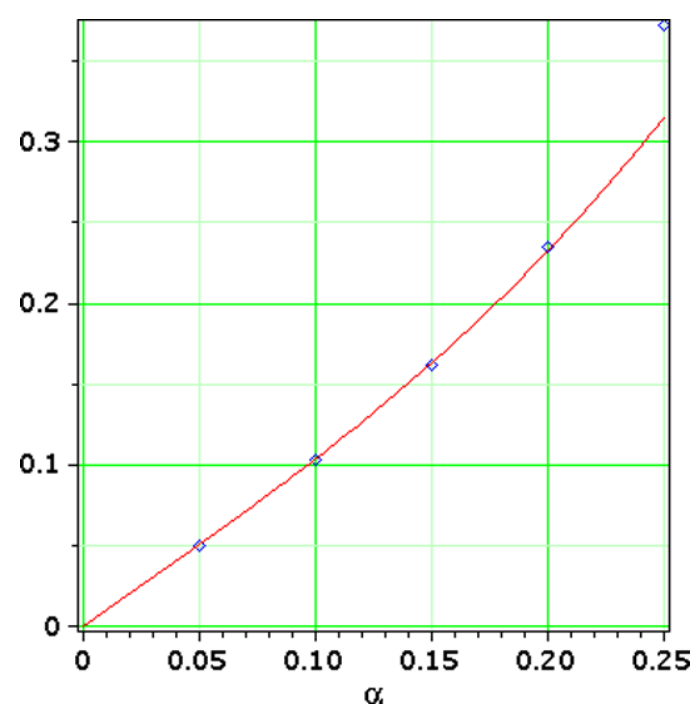

Fig. 9 The coefficients of variation of the beam pulsation frequency

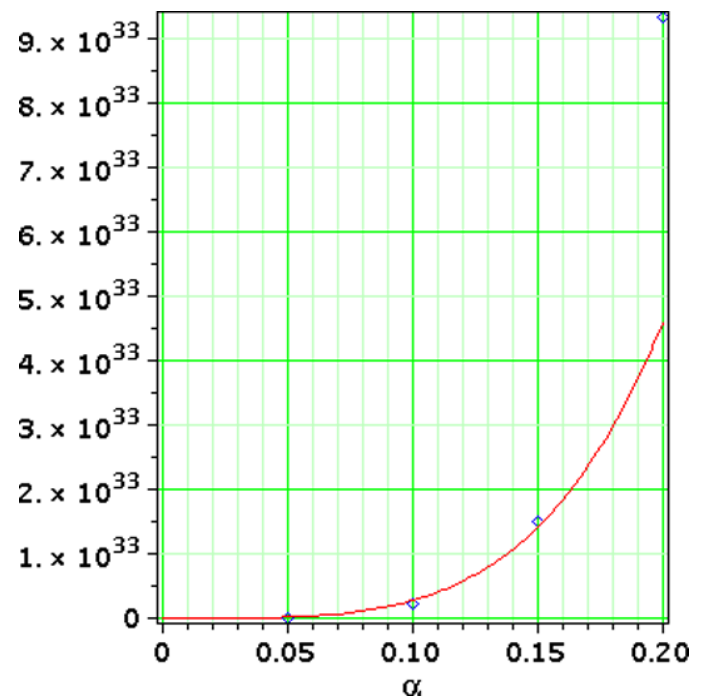

Fig. 10 The third central probabilistic moment of the beam pulsation frequency

the continuous lines, whereas those adequate to the simulations are marked with the diamonds.

The general observation that can be made in Figs. 7-11 is that all the moments and characteristics increase together with the additional increase of the input $\alpha(l)$. The expectations start from the deterministic result for $\alpha(l)=0$, whereas all the remaining quantities begin with 0 , which is also theoretically justified. The second general observation is that the stochastic perturbation approach underestimates the probabilistic

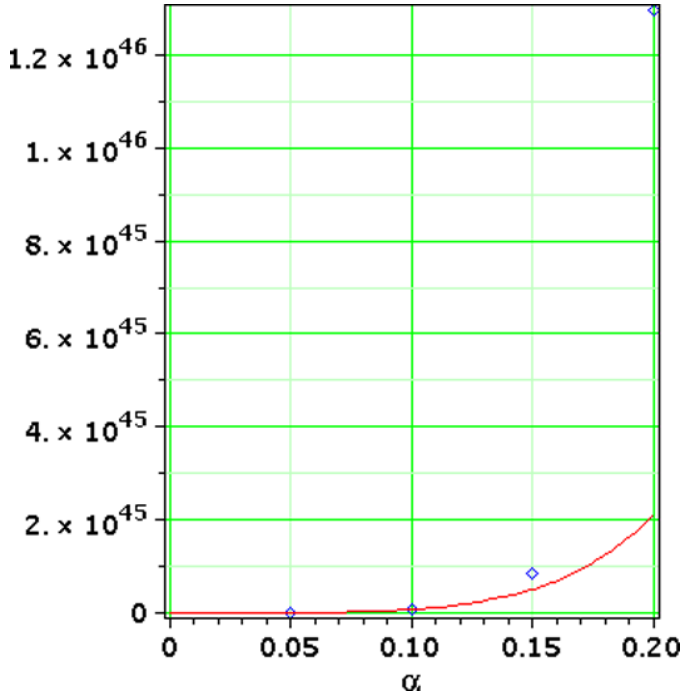

Fig. 11 The fourth central probabilistic moment of the beam pulsation frequency

characteristics having the values always smaller than the additional estimators. Further, one may conclude that the expectations are almost the same in both approaches for $\alpha(l) \in[0.0,0.25]$, the second order characteristics agree well for $\alpha(l) \in[0.0,0.20]$; third and fourth probabilistic moments accuracy demands however $\alpha(l) \in[0.0,0.15]$. Finally, we see that the random dispersion is amplified by this system (cf. Fig. 9) and the amplification ratio increases together with $\alpha(l)$. It is necessary to underline that the consistency of higher probabilistic moments computed using perturbation and simulation techniques may be decisively increased by a significant extension of the Taylor expansions length.

As the example of the polynomial expression including the perturbation parameter $\varepsilon$, the beam length $E[l]$ and its coefficient of variation $\alpha(l)$ we derive the third central probabilistic moment, cf. (9), where the variable $\omega$ replaces the function $T(t, b)$. There holds

$$
\begin{aligned}
\mu_{3}(\omega)= & \frac{15}{8} \varepsilon^{6} \alpha^{6}(l) E^{6}[l]\left(1.358 \times 10^{88} E^{8}[l]\right. \\
& -4.660 \times 10^{81} E^{7}[l]+6.997 \times 10^{74} E^{6}[l] \\
& -6.016 \times 10^{67} E^{5}[l]+3.249 \times 10^{60} E^{4}[l] \\
& -1.134 \times 10^{53} E^{3}[l]+2.515 \times 10^{45} E^{2}[l] \\
& \left.-3.283 \times 10^{37} E[l]+1.975 \times 10^{29}\right)^{3} \\
& +\frac{9}{2} \varepsilon^{4} \alpha^{4}(l) E^{4}[l]\left(1.509 \times 10^{87} E^{9}[l]\right.
\end{aligned}
$$




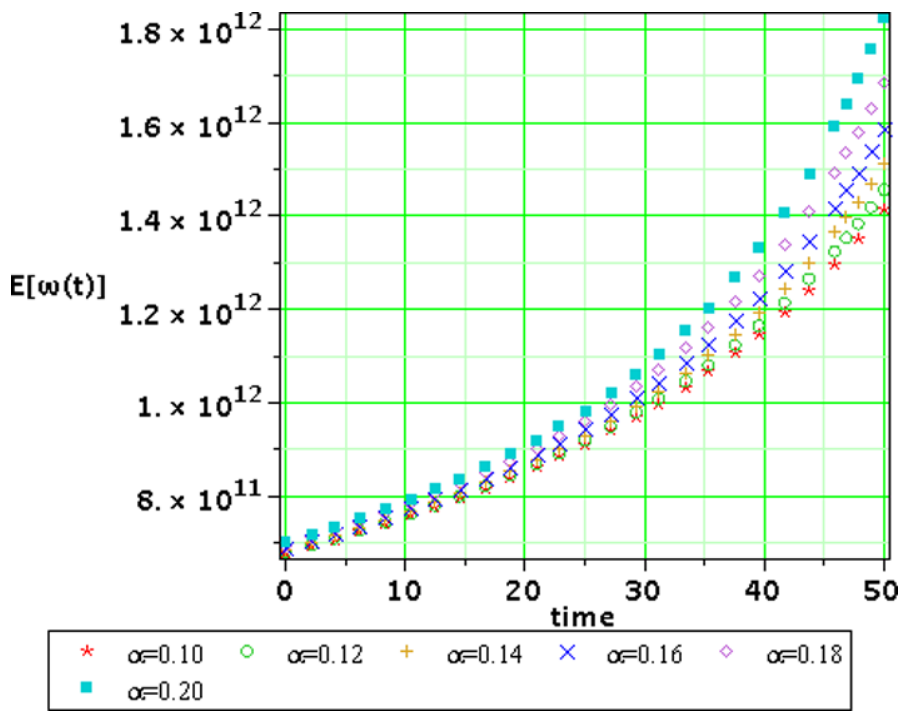

Fig. 12 Time fluctuations for the expected values of the thermo-pulsation

$$
\begin{aligned}
& -5.825 \times 10^{80} E^{8}[l]+9.996 \times 10^{73} E^{7}[l] \\
& -1.003 \times 10^{67} E^{6}[l]+6.498 \times 10^{59} E^{5}[l] \\
& -2.835 \times 10^{52} E^{4}[l]+8.385 \times 10^{44} E^{3}[l] \\
& -1.641 \times 10^{37} E^{2}[l]+1.975 \times 10^{29} E[l] \\
& \left.-1.173 \times 10^{21}\right)^{2}\left(1.358 \times 10^{88} E^{8}[l]\right. \\
& -4.660 \times 10^{81} E^{7}[l]+6.997 \times 10^{74} E^{6}[l] \\
& -6.016 \times 10^{67} E^{5}[l]+3.249 \times 10^{60} E^{4}[l] \\
& -1.134 \times 10^{53} E^{3}[l]+2.515 \times 10^{45} E^{2}[l] \\
& \left.-3.283 \times 10^{37} E[l]+1.975 \times 10^{29}\right) .
\end{aligned}
$$

The particular coefficients accompanying the powers of $E[l]$ comes from the polynomial approximation, of course. Let us note that the most optimal choice for the method order may be done after a comparison of the difference in the particular moments value computed for the increasing orders approximations.

\subsection{Numerical verification of the stochastic ageing}

Now, let us consider the stochastic variations in the piezoelectric beam length using the following linear representation for stochastic variations [6] in its length:

$l(\mathbf{x} ; \omega ; t)=l^{0}(\mathbf{x} ; \omega)-i(\mathbf{x} ; \omega) t$.
The Gaussian random field $l^{0}(\mathbf{x} ; \omega)$ is equivalent to the initial length of the beam, whereas $i(\mathbf{x} ; \omega)$ represents the velocity of ageing process for this parameter. From the mathematical point of view this process is continuous with the continuous time and, which can be relatively easy proven, non-stationary (since the unboundedness of the variance). For the needs of computer simulation performed in MAPLE system we analyze once more the same elastic beam as before and the following additional data are adopted: $E\left[l^{0}(\mathbf{x} ; \omega)\right]=3.9 \times 10^{-8} \mathrm{~m}, E[i(\mathbf{x} ; \omega)]=3.9 \times$ $10^{-10} \mathrm{~m} / \mathrm{yr}$, which is equivalent to $1 \%$ loss of the initial length per a year. As it is documented by Figs. 1215 , the coefficients of variation for this process are taken here as equal to $0.10,0.12,0.14,0.16,0.18$ and 0.20 and the stochastic fluctuations of the thermopulsation eigenvalue are determined for $t \in[0,50 \mathrm{yrs}]$. Consequently we analyze (1) the expected values histories (Fig. 12), (2) the standard deviations time fluctuations (Fig. 13), (3) as well as the asymmetry coefficients and kurtosis histories (Figs. 14 and 15) for the studied eigenvalue.

As it is quite clear, all stochastic properties are strongly affected by the input coefficient of variation for initial value and ageing velocity for the device length. As one could expect from the results obtained in the previous subsection, both expectations and standard deviations increase together with the lifetime of the device, while kurtosis demonstrates quite opposite tendency. The behavior of the asymmetry coefficient 




Fig. 13 Time fluctuations for the standard deviation of the thermo-pulsation

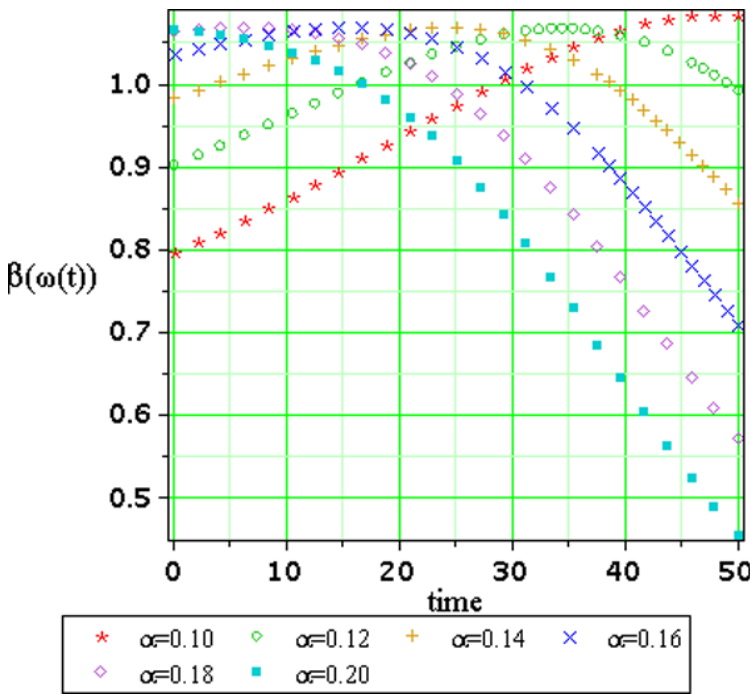

Fig. 14 Time fluctuations for the asymmetry coefficients of the thermo-pulsation

is much more complex-for larger values of input $\alpha$ starts to decrease from the very beginning, whereas for minimum value $(0,10)$ it increases throughout almost entire time domain. The intermediate values of the input coefficient of variation result in initial increase to some local extremum and further decreasing. Let us mention that all those stochastic characteristics are continuous, smooth and differentiable time functions with no local oscillations. Of course, the differences between the expected values histories obtained for different values of input $\alpha$ are not so large as for the standard deviations in the same time moment (cf.



Fig. 15 Time fluctuations for the kurtosis of the thermo-pulsation

Figs. 12 and 13), however those differences do not increase together with the stochastic moment order.

\section{Final remarks}

The derivations presented in this paper show how to apply the generalized $n$th order stochastic perturbation technique for a numerical solution to the coupled thermo-piezoelectric problems using analytical derivations; at the same time we demonstrate the details of the Stochastic Finite Element Method computational 
implementation. This hybrid computational technique may serve efficiently for structural design sensitivity analysis, probabilistic studies of structural behavior due to the stationary uncertainty in some design parameters (or its group with or without correlations) as well as for full stochastic reliability analysis under some non-stationary fluctuations in those parameters. Numerical results obtained in the paper show that the randomization of some design parameters (like the beam length) according to the Gaussian distribution may lead to the non-Gaussian structural response of the system (like thermo-pulsation frequency) and the random dispersion of this output may be significantly larger than the input one. Computational analysis contained above shows that using this method embedded into any computer algebra system one may determine practically any probabilistic characteristics of the output - the only remaining problem is the length of an expansion used to derive the necessary formulas. Quite independently from the perturbation order, this technique seems to be significantly faster than the Monte-Carlo method and allows to derive the analytical expressions for specific random (and also stochastic) output functions (as the only one). A comparison of the methodology presented with the simulation technique shows generally that the perturbation technique underestimates the basic probabilistic moments values. The first two probabilistic moments are computed reliably for $\alpha(l) \in[0.0,0.20]$, whereas next two central moments need smaller values, i.e. $\alpha(l) \in[0.0,0.15]$. Having those probabilistic characteristics determined, any of the existing reliability methods may be used for an effective determination of failure probability (and reliability indices) for the MEMS devices [12] being modeled using this mathematical and numerical apparatus.

Acknowledgements The first author would like to acknowledge the financial support of the Polish Ministry of Science and Higher Education in the framework of the research grant NN 519386 636. The second author wish to thank the Cariplo Foundation for a grant 2009 on the topic "Surface interactions in micro and nanodevices".

Open Access This article is distributed under the terms of the Creative Commons Attribution Noncommercial License which permits any noncommercial use, distribution, and reproduction in any medium, provided the original author(s) and source are credited.

\section{References}

1. Aboudi J (2001) Micromechanical analysis of fully coupled electro-magneto-thermo-elastic multiphase composites. Smart Mater Struct 10:867-877

2. Ardito R, Comi C, Corigliano A, Frangi A (2008) Solid damping in micro-electro-mechanical systems. Meccanica 43:419-428

3. Bednarcyk BA (2002) A fully coupled micro/macro theory for thermo-electro-magneto-elasto-plastic composite laminates. Report NASA/CR-2002-211468, Ohio Aerospace Institute

4. Carman GP, Cheung K-S, Wang D (1995) Micromechanical model of a composite containing a conservative nonlinear electro-magneto-thermo-mechanical material. J Intell Mater Syst Struct 6(5):691-699

5. Gao Y, Xu B, Huh H (2010) Electromagneto-thermomechanical behaviors of conductive circular plate subject to time-dependent magnetic fields. Acta Mech 210(1-2):99-116

6. Kamiński M (2008) Homogenization of the fiber-reinforced composites under stochastic ageing processes. Int J Multiscale Comput Eng 64:361-370

7. Kamiński M (2010) Potential problems with random parameters by the generalized perturbation-based stochastic finite element method. Comput Struct 88:437-445

8. Kleiber M, Hien TD (1992) The stochastic finite element method. Wiley, Chichester

9. Kumar A, Chakraborty D (2009) Effective properties of thermo-electro-mechanically coupled piezoelectric fiber reinforced composites. Int J Mater Des 30(4):1216-1222

10. Lepage D (2006) Stochastic finite element method for the modeling of thermoelastic damping in micro-resonators. $\mathrm{PhD}$ Dissertation, University of Liege

11. Li JY (2003) Uniqueness and reciprocity theorems for linear thermo-electro-magneto-elasticity. Q J Mech Appl Math 56(1):35-43

12. Mariani S, Ghisi A, Fachin F, Cacchione F, Corigliano A, Zerbini S (2008) A three-scale FE approach to reliability analysis of MEMS sensors subject to impacts. Meccanica 43:469-483

13. Nowacki W (1975) Thermodiffusion in solids. J Theor Appl Mech 13(2):143-158

14. Pérez-Fernández LD, Bravo-Castillero J, RodriguezRamos R, Sabina FJ (2009) On the constitutive relations and energy potentials of linear thermo-magneto-electroelasticity. Mech Res Commun 36(3):343-350

15. Tan P, Tong L (2002) Modeling for the electro-magnetothermo-elastic properties of piezoelectric-magnetic fiber reinforced composites. Composites, Part A, Appl Sci Manuf 33(5):631-645

16. Tong ZH, Lo SH, Jiang CP, Cheung YK (2008) An exact solution for the three-phase thermo-electromagneto-elastic cylinder model and its application to piezoelectric-magnetic fiber composites. Int J Solids Struct 45(20):5205-5219 